\title{
Interfacial Properties of Polymeric Liquids
}

\author{
Miguel Aubouy, ${ }^{1}$ Manoel Manghi, ${ }^{1}$ and Elie Raphaël ${ }^{2}$ \\ ${ }^{1}$ Service des Interfaces et des Matériaux Moléculaires et Macromoléculaires, ${ }^{*}$ D.R.F.M.C., CEA-Grenoble, \\ 38054 Grenoble cedex 9, France \\ ${ }^{2}$ Laboratoire de Physique de la Matière Condensée, ${ }^{\dagger}$ Collège of France, 75231 Paris cedex 5, France
}

(Received 22 February 2000)

\begin{abstract}
We show that the variations of the surface tension of polymeric solutions $\gamma$ with the molecular weight of the sample $M_{n}$, the temperature $T$, and the volume fraction of monomers $\phi_{b}$ are explained by the self-organization of the long flexible chains that are present in the immediate vicinity of the interface. Within a scaling functional theory we find a simple law $\gamma\left(\phi_{b}, M_{n}, T\right)$ which accounts quantitatively for the experimental data available in the literature with a very high accuracy.
\end{abstract}

PACS numbers: $61.25 . \mathrm{Hq}$

Concentrated polymer solutions made of long flexible chains belong to complex liquids because their properties are dominated by the self-organization of the chains. This is especially relevant at an interface, where the presence of a sharp boundary condition modifies the threedimensional structure of the fluid and induces the presence of a self-structured inhomogeneous layer of chains. This layer is responsible for the interfacial properties of the liquid which can differ from the corresponding properties in the bulk [1]. Most significantly, the interfacial tension of the polymeric phase $\gamma$ is a function of the molecular weight (MW) $M_{n}$ of the macromolecules. In many applications of polymeric materials (coating, adhesion, colloidal stabilization, etc.), $\gamma$ is a crucial parameter, and this feature has important consequences. For polydisperse samples, e.g., a fraction of the chains will adsorb preferentially and modify the composition of the interface in comparison to that of the bulk. Polymeric liquids play a special role among complex liquids, not only because they are widely spread in the industry, but also because they have been studied at a fundamental level for many years now. It is clear that understanding the interfacial properties of these will bring new, broader insights into the interfacial properties of soft condensed matter in general. In this Letter, we present the first theory for the surface tension of polymeric solutions which is able to reproduce the variations of $\gamma$ with MW, temperature, $T$, and volume fraction of monomers, $\phi_{b}$.

The variations of $\gamma$ with MW for molten polymer liquids, $\gamma\left(\phi_{b}=1, M_{n}, T\right)$, have received much experimental attention. Data are usually fitted by the empirical law $\gamma=\gamma_{\infty}-k / M_{n}^{v}$, with $v=\frac{2}{3}$ and $v=1$ for low MW and high MW samples, respectively [2]. In the theoretical literature, one may find three different approaches to explain the empirical law: (i) Dee and Sauer were able to fit the experimental data with few parameters through an empirical equation of state for the bulk phase [2]. However, the overall treatment needed to reach an agreement remains quite complicated. (ii) Using scaling arguments based on the idea of a specific tensioactivity of the chain ends, de Gennes recovers the empirical equation, but with $v=\frac{1}{2}$ [3]. (iii) Sophisticated self-consistent field (SCF) approaches yield $v=1$ [4-7], and thus lead to no improvement. Furthermore, $\gamma$ is predicted to be an increasing function of $T$, unlike what is reported in the experimental literature. Much less is known about semidilute solutions $\left(\phi_{b}^{*} \leq \phi_{b} \leq 1\right)$. To the best of our knowledge, we know only about a modified Cahn-Hilliard theory [8] which yields $\Delta \gamma \sim-\phi_{b}^{5 / 4}$ (good solvent), with no MW dependence. However, this conclusion differs from the experimental observations $[9,10]$.

Here, we estimate $\gamma\left(\phi_{b}, M_{n}, T\right)$ using a scaling functional theory (SFT) initially proposed to describe the structure of adsorbed polymer layers [11]. This approach is a modern (simplified) version of an early theory by Hoeve, Silberberg, and Di Marzio where the interface is described as a thermodynamical ensemble of loops and tails very polydispersive in size $[12,13]$. The structure of the layer in the immediate vicinity of the interface results from the competition between (a) the interactions between the chain segments: two-body interactions (good solvent) or stretching (melt); (b) the entropy associated to the large polydispersity in size of the loops that are formed at the interface; and (c) a specific attraction of chain ends towards the interface. This entropy (b) was not considered in the previous theories for the surface tension of a polymeric solution. On general grounds, we expect that it contributes to $\gamma$ : The longer the chains are, the wider is the range of loop sizes accessible for adsorbed loops, and thus the larger is the entropy of the interface. Our theory which combines the three contributions yields a simple law $\gamma\left(\phi_{b}, M_{n}, T\right)$ which accounts quantitatively for the experimental data. This law is first estimated in the melt limit, and then generalized to an arbitrary volume fraction.

In what follows, the melt is a set of linear, neutral, homogeneous, flexible, monodisperse chains of $N$ monomers of size $a . \quad\left(M_{n}=N M_{n 0}\right.$, where $M_{n 0}$ is the MW of the monomer.) We assume that the air/melt interface is sharp, and $\phi_{b}=1$. We distinguish between those chains which have at least a monomer in direct contact with the surface, 
"adsorbed" chains, and the others, "free" chains. Adsorbed chains form loops and tails of various sizes. Trains (chain segments lying on the surface) are considered as a succession of "loops of two monomers." In the spirit of Ref. [11], we make the following simplifications: (i) Each loop of $2 n$ monomers is considered as two "pseudotails" of length $n$. In other words, we describe the interface as a thermodynamical set of tails, polydisperse in size, end attached to the surface. (ii) The tails behave identically: Their conformation is described by a single function $n(z)$, where $n$ is the curvilinear index along the tail, and $z$ is the distance to the surface $[n(a)=1]$. (iii) The free chains do not penetrate the layer formed by the adsorbed chains.

Our main tool is the "loop size profile," $S$, such that $S(n)=a^{-2} \int_{n}^{N} P(u) d u$, where $P$ is the statistical distribution of tail sizes in monomer units. The free energy (per $\mathrm{cm}^{2}$ ) of the adsorbed chains may be written as [11]

$$
\bar{F}_{\text {ads }}\{S\} \cong \gamma_{0}+\frac{k_{B} T}{a^{2}} \int_{1}^{N}\left\{k_{1}\left[a^{2} S(n)\right]^{3}+\left[-a^{2} S^{\prime}(n)\right] \ln \left[-a^{2} S^{\prime}(n)\right]-\frac{2 \Delta}{N}\left[a^{2} S(n)\right]\right\} d n,
$$

where $k_{1} \sim 1$ is a constant, $k_{B} T$ is the thermal energy, and $S^{\prime}=\partial S / \partial n$. The quantity $\gamma_{0}$ is the van der Waals energy (per $\mathrm{cm}^{2}$ ), which accounts for monomer/air interactions. The second term in the right-hand side (rhs) of Eq. (1) is the elastic energy per unit surface: $\int S(n)(d z / d n)^{2} d n$ rewritten using the local conservation of monomers: $d z / d n=a^{3} S(n)$. The third term is the usual entropy associated with a set of polydisperse objects. The last term accounts for chain-ends attraction. This attraction arises because the last monomers have a polarizability slightly different from that of a monomer in the middle of the backbone [14]. The number (per $\mathrm{cm}^{2}$ ) of adsorbed chains is $\Gamma / N=\int S(n) d n / N$. Assuming an attractive energy $\Delta k_{B} T$ per chain ends in direct contact with the surface, and two adsorbed ends per polymer, this gives a contribution $-(2 \Delta / N) S(n)$ to the functional. We expect that $\Delta$ should be of order unity and the third term in Eq. (1) does not contribute to the equilibrium profile. This argument can be made quantitative. Suppose $\Delta=0$, minimizing Eq. (1) yields the equilibrium loop-size profile: $S_{\text {eq }}(n) \cong$ $\left(2 k_{1}\right)^{-1 / 2} /\left(a^{2} n^{1 / 2}\right) \quad(1 \leq n \leq N)$. Our approximation is valid whenever $a^{4} S_{\text {eq }}(n)^{3} \gg(2 \Delta / N) S_{\text {eq }}(n)$, or $n \ll N /\left(4 k_{1} \Delta\right)$ in the domain of integration. If $\Delta$ is large, the solution $S_{\mathrm{eq}}(n) \sim n^{-1 / 2}$ is not valid. In this strong adsorption regime, the adsorbed chains do not keep their Gaussian shape: They stretch away from the interface to increase the number of adsorbed ends [11]. However, for small values of $\Delta$ (weak adsorption regime), $N /\left(4 k_{1} \Delta\right) \gg$ $N$, the layer is not affected by this contribution to the free energy. In physical terms, this means that there is a range of values $0 \leq \Delta \leqq 1 / 4 k_{1}$ where all the chain ends are captured by the surface without disturbing the Gaussian shape of the chains. In practice, there is a further restriction to our model [3]: At very low $\Delta$, only a fraction of ends are captured. This means that Eq. (1) is valid only for values of $\Delta$ close to $1 / 4 k_{1}$. In what follows, we will assume that both conditions are fulfilled, and we will use Eq. (1) together with the solution $S_{\text {eq }}(n) \sim$ $n^{-1 / 2}$. The extension of the layer can then be estimated: $H=\int S_{\text {eq }} d n \cong a N^{1 / 2}$.

Let us now calculate the surface tension. The quantity $\gamma$ is the penalty for the presence of the free surface: $\gamma=\bar{F}_{\text {ads }}\left\{S_{\text {eq }}\right\}-\bar{F}_{\text {bulk }}$, where $\bar{F}_{\text {bulk }}$ is the free energy of our reference state, the bulk of the melt. The quan- tity $\bar{F}_{\text {bulk }}$ has only one contribution: the elastic "energy" of Gaussian chains. Counting $k_{B} T$ per chain, we find $\bar{F}_{\text {bulk }} /\left(k_{B} T / a^{2}\right) \cong(H / a) / N \cong N^{-1 / 2}$. Then, $\gamma$ can be computed, yielding

$$
\gamma\left(\phi_{b}=1, M_{n}, T\right)=\gamma_{\infty}+D M_{n 0}^{1 / 2} \frac{k_{B} T}{a^{2}} \frac{\ln \left(M_{n} / M_{n}^{*}\right)}{M_{n}^{1 / 2}},
$$

where $\gamma_{\infty}=\gamma_{0}-C\left[\left(k_{B} T\right) / a^{2}\right] . \quad$ The quantity $M_{n}^{*} \cong B e^{8 / 3 \Delta} M_{n 0}$ which appears in Eq. (2) plays the role of a characteristic MW of the polymer under consideration. With reasonable values $(\Delta \cong 1)$, we find $N^{*}=M_{n}^{*} / M_{n 0} \sim 50$. (B, $C$, and $D$ are numerical coefficients of order unity.) Qualitatively, Eq. (2) is in very good agreement with the empirical law which has been proposed. Indeed, when $M_{n}<M_{n}^{*}$, Eq. (2) is equivalent to $\gamma=\gamma_{\infty}-k / M_{n}^{v}$ with an apparent exponent $v$, which is both larger than $\frac{1}{2}$ and an increasing function of the MW. Concerning the dependence with temperature, it is found that $\partial \gamma / \partial T$ is (a) negative, (b) of the order of $k_{B} / a^{2}$, (c) almost independent of the particular polymer studied, and (d) a function of the MW of the sample [1]. This is what we find with Eq. (2).

It is simple to generalize our findings to the semidilute regime (good solvent) in the limit where the monomers are adsorbed at the interface [15]. This involves two steps. First, $S_{\mathrm{eq}}$ has to be renormalized: $1 /\left(a^{2} n^{1 / 2}\right) \rightarrow$ $1 /\left\{\xi^{2}\left[n /(\xi / a)^{5 / 3}\right]^{1 / 2}\right\}$, where $\xi \cong a \phi_{b}^{-3 / 4}$ is the "blob" size [16]. Thus $S_{\text {eq }}(n) \cong \phi_{b}^{7 / 8} /\left(a^{2} n^{1 / 2}\right)$. Second, we have to account for the increase of the volume fraction in the immediate vicinity of the surface. The bulk chemical potential per monomer is $k_{B} T \phi_{b}^{5 / 4}$. Assuming that $\phi(z) \cong(z / a)^{-4 / 3}$, as is expected in the dilute limit [8], we find an extra contribution $\left[\left(k_{B} T\right) / a^{2}\right] \phi_{b}^{5 / 4} \int_{a}^{\xi} \phi(z) d z \cong$ $-\left[\left(k_{B} T\right) / a^{2}\right] \phi_{b}^{5 / 4}$ to $\gamma$. After some calculations, we find

$$
\begin{aligned}
\Delta \gamma\left(\phi_{b}, M_{n}, T\right) \cong & -E \frac{k_{B} T}{a^{2}} \phi_{b}^{5 / 4} \\
& +D M_{n 0}^{1 / 2} \frac{k_{B} T}{a^{2}} \frac{\phi_{b}^{7 / 8}}{M_{n}^{1 / 2}} \ln \left(\frac{M_{n}}{\phi_{b}^{7 / 12} M_{n}^{*}}\right),
\end{aligned}
$$

where $E \sim 1$ is a numerical coefficient. 


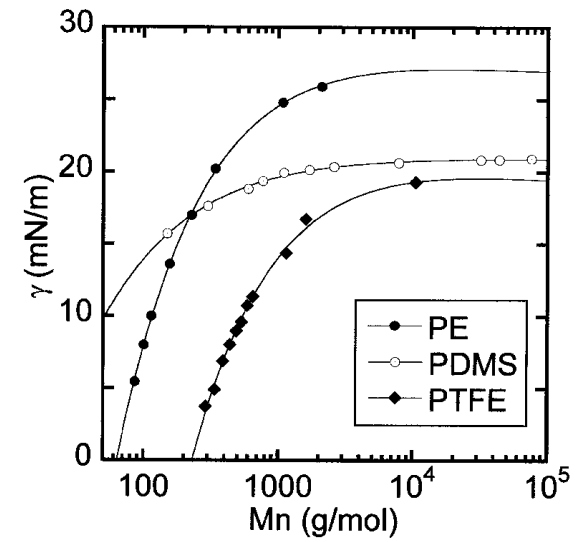

FIG. 1. Surface tension of molten polymer solutions $\left(\phi_{b}=1\right)$ vs MW for three different polymers: PE at $150{ }^{\circ} \mathrm{C}$ [17], PDMS at $20^{\circ} \mathrm{C}$ [18], and PTFE at $100{ }^{\circ} \mathrm{C}$ [19]. The solid lines correspond to the theoretical law [Eq. (2)] fitted with two adjustable parameters $\left(D, N^{*}\right)$. See text and Table I for details.

Although we are working at a scaling level, we have performed a quantitative comparison with the data. Figure 1 displays the surface tension $\gamma\left(M_{n}\right)$ for three different polymers: polyethylene (PE), poly(dimethylsiloxane) (PDMS), and poly(tetrafluoroethylene) (PTFE). We have taken $\gamma_{\infty}=\gamma_{0}+T \partial \gamma /\left.\partial T\right|_{M_{n} \rightarrow \infty}$ as given by the experimental data (Table I). The data have thus been fitted with Eq. (2) with two adjustable parameters $\left(D, N^{*}\right.$, see Table I). The quantitative agreement is remarkably good. For a given polymer, the validity of Eq. (2) can also be tested by varying the temperature. Figure 2 displays experimental data for PDMS at two different temperatures. Inserted in Fig. 2, we display two different series of experiments on PDMS, where the variations $\gamma(T)$ at fixed $M_{n}$ have been measured. In both cases, the solid lines represent Eq. (2) with the values of $N^{*}$ and $D$ that we have found previously, i.e., with no adjustable parameters this time. Figure 3 displays the variations of $\gamma\left(\phi_{b}\right)$ for semidilute solutions of PDMS of different MW. Most significantly, we observe a splay of the different curves when $\phi_{b}$ increases, indicating that $\gamma$ is not simply the sum of a pure MW and a pure $\phi_{b}$ contribution. This splay is well accounted for by the second term of the rhs of Eq. (3) with no adjustable parameter. The value of $E=0.46 \pm 0.01$ is determined once for a given MW. The quantitative agreement with data is again very good within, plausibly, a systematic experimental uncertainty.

We have spent some time in collecting and fitting the experimental data for two reasons. The first reason is to see whether our predictions would represent an improvement upon the different theories proposal before. The second reason is to check whether our predictions are an artifact of our theory. There is an important conceptual difficulty which is not explicit. Suppose $\gamma_{0}=0$ and $\Delta=0$, and consider a piece of melt divided into two semi-infinite spaces: in case (A) the separating surface is impenetrable; in case (B) the dividing plane is imaginary. Obviously, there is no interfacial tension in case
TABLE I. Experimental values of the quantities $a, M_{n 0}$ [20], $\gamma_{0}$, and $-\partial \gamma /\left.\partial T\right|_{M_{n} \rightarrow \infty}$ [1] for PDMS, PE, and PTFE. These, together with the fits, allow a precise determination of $D$ and $N^{*}$

\begin{tabular}{lccc}
\hline \hline & PE & PDMS & PTFE \\
\hline$a(\mathrm{~nm})$ & 1.08 & 0.73 & 3.7 \\
$M_{n 0}(\mathrm{~g} / \mathrm{mol})$ & 98 & 185 & 2400 \\
$\gamma_{0}(\mathrm{mN} / \mathrm{m})$ & 53.71 & 37.78 & 43.96 \\
$-\partial \gamma /\left.\partial T\right|_{M_{n} \rightarrow \infty}$ & & & \\
$\quad\left(10^{-2} \mathrm{mN} / \mathrm{m} / \mathrm{K}\right)$ & 6.187 & 5.786 & 6.377 \\
$D$ & $0.85 \pm 0.04$ & $0.14 \pm 0.03$ & $5.6 \pm 0.02$ \\
$N^{*}$ & $27 \pm 3$ & $95 \pm 5$ & $2 \pm 1$ \\
\hline \hline
\end{tabular}

(B). We find a tension in case (A) which has two origins. Let us call (c), the monomers in contact with the plane, and (n), the others. Because the segments have to go back to the melt, each monomer (c) loses an orientational choice. This is the contribution $k_{B} T \int S_{\mathrm{eq}}^{3} d n \cong k_{B} T / a^{2}$. We find a second contribution which we think is related to a broken symmetry. Because they experience a local force due to the impenetrable wall, (c) monomers are different. They become distinguishable. As we see it, the situation is quite similar to the Gibbs paradox. In case (B), only (n) monomers are present: There is no physical reason why we should distinguish those monomers which touch the imaginary plane from the others. In case (A), we may distinguish two types of monomers, as if we were in the presence of two different chemical species (c) and (n). Then, an entropy of mixing should be accounted for this difference. This is in our theory the origin of the contribution $k_{B} T \int-S_{\mathrm{eq}}^{\prime} \ln \left(-S_{\mathrm{eq}}^{\prime}\right) d n \sim k_{B} T / a^{2}(\ln N) / N^{1 / 2}$ to $\gamma$.

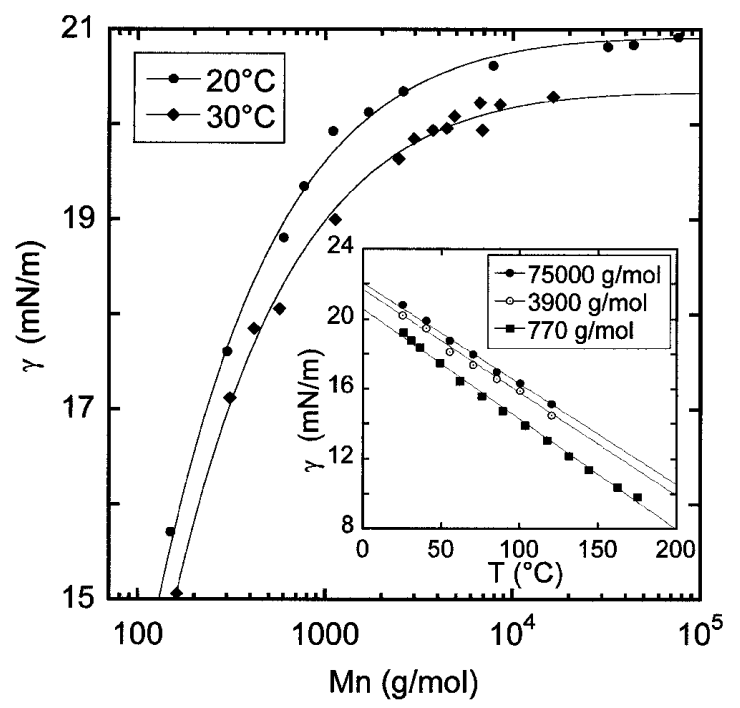

FIG. 2. Effect of temperature on the surface tension of molten polymer solutions $\left(\phi_{b}=1\right)$. $\gamma$ versus $M_{n}$ for PDMS at $T=$ $20{ }^{\circ} \mathrm{C}$ (data taken from [18]) and $T=30{ }^{\circ} \mathrm{C}$ [21]. The inset displays the variations $\gamma(T)$ at fixed $M_{n}$ for three different values of the MW: $770 \mathrm{~g} / \mathrm{mol}$ (data taken from [22]), 3900 and $75000 \mathrm{~g} / \mathrm{mol}$ [23]. The solid line is the theoretical law [Eq. (2)] with no adjustable parameter. 


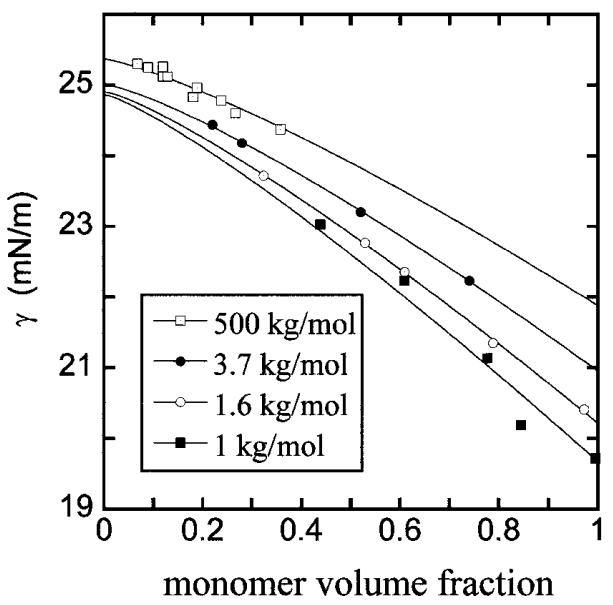

FIG. 3. Surface tension of semidilute solutions of PDMS in toluene vs the monomer volume fraction, $\phi_{b}$, for several different MW. The different symbols correspond to experimental data of [9] $\left(T=24{ }^{\circ} \mathrm{C}, M_{n}: 500 \mathrm{~kg} / \mathrm{mol}\right)$ and [10] $\left(T=19.5^{\circ} \mathrm{C}\right.$, $M_{n}: 1 \mathrm{~kg} / \mathrm{mol}, 1.6 \mathrm{~kg} / \mathrm{mol}, 3.7 \mathrm{~kg} / \mathrm{mol}$ ). Full lines are Eq. (3) (see text for details).

A careful comparison of our SFT approach with SCF theories is out of the scope of this Letter and will be done elsewhere [15]. We speculate that this second contribution to $\gamma$ is lost in a description where the only relevant field, $\phi(z)$, does not account for chain connectivity. This would explain why we get only the next to leading order contribution $\left(\Delta \gamma \sim N^{-1}\right)$ with a SCF theory.

Our model involves several simplifications. A simple calculation shows that cutting a Gaussian loop of $n$ monomers into two symmetrical, independent, grafted tails amounts to $\Delta F_{\text {cut }} \cong k_{B} T\left(\frac{3}{2} \ln n-\alpha / n+\right.$ $\beta)$ per loop $(\alpha, \beta \sim 1)$. We expect an error $\Delta F_{\text {ads }} \sim$ $\int\left(-1 / 2 S_{\text {eq }}^{\prime}\right) \Delta F_{\text {cut }} d n$, which can be rewritten as $-\frac{1}{2} \int-S_{\text {eq }}^{\prime} \ln \left(-S_{\text {eq }}^{\prime}\right) d n+O\left(N^{-1 / 2}\right)$. Because this error does not bring any new leading contribution (and does not cancel any of the terms) in Eq. (2), we are confident that hypothesis (i) does not modify our essential results (see also [11]). Once this is assumed, hypothesis (ii) is reasonable and has been used in SCF theories of the same problem [24]. Relaxing hypothesis (iii) would certainly modify $S_{\text {eq }}$, but we do not expect that the scaling law will be affected. A direct confirmation of that point is provided by experiments where $S$ is measured after the free chains have been removed [25]. We have also assumed an incompressible melt ( $a=$ const), as is usual in first approximation [3-7]. This may not always be the case [26]. Of course, the air/liquid interface is a rather restricted situation. Equations (2) and (3) hold for mixtures of polymers and colloidal solids, and for blends of polymers in the strong segregation limit.

We are grateful to Pierre-Gilles de Gennes, Mohamed Daoud, Sanat Kumar, and Jeff Koberstein for useful discussions. We thank Jean-François Legrand, Thomas Waigh, and Avi Halperin for a critical reading of the manuscript.

*U.M.R. No. 5819: C.E.A., C.N.R.S., and Université Joseph-Fourier.

${ }^{\dagger}$ U.R.A. No. 792 du CNRS.

[1] S. Wu, Polymer Interfaces and Adhesion (Dekker, New York, 1982).

[2] G. T. Dee and B. Sauer, Adv. Phys. 47, 161 (1998).

[3] P.-G. de Gennes, C. R. Acad. Sci. II (Paris) 307, 1841 (1988).

[4] D. T. Wu et al., J. Polym. Sci. B, Polym. Phys. 33, 2373 (1995).

[5] E. Helfand, S. M. Bhattacharjee, and G.H. Fredrickson, J. Chem. Phys. 91, 7200 (1989).

[6] H. Tang and K. F. Freed, J. Chem. Phys. 94, 6307 (1991).

[7] A. V. Ermoshkin and A. N. Semenov, Macromolecules 29, 6294 (1996).

[8] P.-G. de Gennes, Macromolecules 14, 1637 (1981).

[9] J. L. Gaines, J. Phys. Chem. 73, 3143 (1969).

[10] R. Ober, L. Paz, C. Taupin, and P. Pincus, Macromolecules 16, 50 (1983).

[11] M. Aubouy, O. Guiselin, and E. Raphaël, Macromolecules 29, 7261 (1996).

[12] C. A. J. Hoeve, E. A. Di Marzio, and P. Peyser, J. Chem. Phys. 42, 2558 (1965); A. Silberberg, J. Chem. Phys. 48, 2835 (1968).

[13] O. Guiselin, Europhys. Lett. 17, 225 (1992).

[14] W. Zhao et al., Macromolecules 26, 561 (1993).

[15] M. Manghi and M. Aubouy, Macromolecules (to be published).

[16] P.-G. de Gennes, Scaling Concepts in Polymer Physics (Cornell University Press, Ithaca, 1985), second printing.

[17] B. Sauer and G. T. Dee, J. Colloid Interface Sci. 162, 25 (1994).

[18] B. Sauer and G. T. Dee, Macromolecules 24, 2124 (1991).

[19] B. Sauer and G. T. Dee, Macromolecules 27, 6112 (1994).

[20] S. M. Aharoni, Macromolecules 16, 1722 (1983).

[21] C. Jalbert et al., Macromolecules 26, 3069 (1993).

[22] G. T. Dee and B. Sauer, J. Colloid Interface Sci. 152, 85 (1992).

[23] Q. S. Bhatia et al., J. Colloid Interface Sci. 106, 353 (1985).

[24] S. Milner, T. Witten, and M. Cates, Macromolecules 22, 853 (1989).

[25] L. Léger, E. Raphaël, and H. Hervet, Adv. Polym. Sci. 138, 185 (1999).

[26] A. Hariharan, S. K. Kumar, and T.P. Russell, Macromolecules 23, 3584 (1990). 